Carlos de Lamare Bastian Pinto

Modelagem de Opções Reais com Processos de Reversão à Média em Tempo Discreto: Uma Aplicação na Indústria Brasileira de Etanol

Tese de Doutorado

Tese apresentada ao Programa de Pós-Graduação em Administração de Empresas da PUC-Rio como requisito parcial para obtenção do título de Doutor em Administração de Empresas.

Orientador: Luiz Eduardo Teixeira Brandão

Rio de Janeiro

Dezembro de 2009 


\section{Modelagem de Opções Reais com Processos de Reversão à Média em Tempo Discreto: Uma Aplicação na Indústria Brasileira de Etanol}

Tese apresentada ao Programa de Pós-Graduação em Administração de Empresas da PUC-Rio como requisito parcial para obtenção do título de Doutor em Administração de Empresas da PUC-Rio. Aprovada pela Comissão Examinadora abaixo assinada.

Prof. Luiz Eduardo Teixeira Brandão Orientador Departamento de Administração - PUC-Rio

Prof. Antonio Carlos Figueiredo Pinto Departamento de Administração - PUC-Rio

Prof. Walter Lee Ness, Jr. Departamento de Administração - PUC-Rio

Prof. Roberto Marcos da Silva Montezano IBMEC

Prof. Diógenes Manoel Leiva Martin Universidade Presbiteriana Mackenzie PPGA- CCSA

Prof. Nizar Messari

Vice-Decano de Pós-Graduação do CCS

Rio de Janeiro, 18 de Dezembro de 2009 
Todos os direitos reservados. É proibida a reprodução total ou parcial do trabalho sem autorização da universidade, do autor e do orientador.

\section{Carlos de Lamare Bastian Pinto}

Graduado em Engenharia Mecânica pela Pontifícia Universidade Católica do Rio de Janeiro (1982), MBA em Gestão de Negócios pelo Ibmec Rio de Janeiro (2001) e Mestrado Profissionalizante em Administração pelo Ibmec Rio de Janeiro (2004), com foco em Avaliação de Empresas e Projetos pela Metodologia de Opções Reais. Profissionalmente possui experiência em Análise Econômica e Financeira de Empresas, Orçamento de Capital (valuation), Business Plan e Desenvolvimento e Modelagem Estratégica de Novos Negócios. Atuou por 20 anos em diversas áreas tais como: TI, Sistemas de Apoio à Gestão, Logística, Energia, Petroquímica, Oil\&Gas, etc. tanto como Consultor Independente, assim como empregado direto em empresas como Vale do Rio Doce.

\section{Ficha Catalográfica}

\section{Pinto, Carlos de Lamare Bastian}

Modelagem de opções reais com processos de reversão à média em tempo discreto: uma aplicação na indústria brasileira de etanol / Carlos de Lamare Bastian Pinto ; orientador: Luiz Eduardo Teixeira Brandão. - 2009. 164 f. : il. (color.) ; $30 \mathrm{~cm}$

Tese (Doutorado em Administração)-Pontifícia Universidade Católica do Rio de Janeiro, Rio de Janeiro, 2009.

Inclui bibliografia

1. Administração - Teses. 2. Opções reais. 3. Processos estocásticos. 4. Reversão à média. 5. Árvore binomial. 6. Árvore bi-variável. 7. Processos estocásticos de dois fatores. 8. Bio-combustíveis. 9. Indústria de etanol. I. Brandão, Luiz Eduardo Teixeira. II. Pontifícia Universidade Católica do Rio de Janeiro. Departamento de Administração. III. Título. 


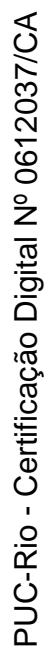

A meus filhos Luiz e Bernardo. 


\section{Agradecimentos}

Ao meu orientador, professor Luiz Eduardo Teixeira Brandão, pelo permanente incentivo e estimulo desde o início deste trabalho.

Aos professores do IAG, em especial Antônio Carlos Figueiredo, Leonardo Lima Gomes, Jorge Ferreira e Walter Ness, pelo apoio nestes anos de doutorado.

Aos professores Roberto Marcos da Silva Montezano e Diógenes Manoel Leiva Martin, pelos comentários e contribuições na defesa.

Ao professor Marco Antônio Guimarães Dias pelas aulas ministradas e pela inspiração.

Às minhas colegas de doutorado, que hoje são minhas grandes amigas, Graziela Fortunado e Marta Dalbem, pelo convívio nestes anos de estudo.

Aos funcionários do IAG, em especial Teresa Campos por sempre estar disposta em ajudar nos assuntos administrativos.

À PUC-Rio, pelo auxílio e pelo ambiente acadêmico.

À minha querida família, meus filhos Luiz e Bernardo, minha esposa Frederika e meus pais Luiz e Célia, sem o amor dos quais nunca teria chegado tão longe.

E, acima de tudo, a Deus por estar presente em todo instante da minha vida. 


\section{Resumo}

Bastian Pinto, Carlos de Lamare; Brandão, Luiz Eduardo Teixeira. Modelagem de Opções Reais com Processos de Reversão à Média em Tempo Discreto: Uma Aplicação na Indústria Brasileira de Etanol. Rio de Janeiro, Tese de Doutorado, p. 164 - Departamento de Administração, Pontifícia Universidade Católica do Rio de Janeiro

A presente tese trata da modelagem por Reversão à Média de incertezas estocásticas, e sua aplicação em opções reais. A utilização de processos estocásticos que não o caminho aleatório, ou Movimento Geométrico Browniano (MGB), geralmente não permitem a utilização de soluções analíticas fechadas para avaliar ativos contingenciais e então são usadas abordagens numéricas discretas. A tese está dividida em três capítulos que cobrem o tema. No primeiro após uma discussão sobre a validação do processo a ser usado são apresentados os modelos mais conhecidos para Movimentos de Reversão à Média (MRM): quatro de fator único, entre eles um aritmético e três geométricos, e mais um de dois fatores. Para todos são mostrados ou desenvolvidos, os processos de discretização, a expressão do valor esperado assim como a estimação de parâmetros a partir de séries históricas. A relevância desse último ponto é devida ao fato que geralmente somente séries históricas são conhecidas para as variáveis incertas nas aplicações de opções reais. O capítulo apresenta ainda o levantamento de parâmetros para os principais modelos apresentados no caso de séries históricas de preços reais de açúcar e etanol pagos ao produtor no Estado de São Paulo. O segundo capítulo trata da modelagem por árvore binomial como método numérico discreto para aproximação de processos de reversão à média. Esta aproximação permite a avaliação de ativos contingentes escritos sobre uma variável cujo valor tenha esse comportamento estocástico. Essa abordagem clássica é usada em inúmeros trabalhos e tem origem na metodologia desenvolvida por Cox, Ross e Rubinstein (1979), a qual só é aplicável a variáveis que tenham um comportamento aproximado por um MGB, excluindo toda uma gama de ativos cujo comportamento é mais bem aproximado por um processo auto regressivo. São 
demonstradas duas formas de aproximação binomial para reversão à média. Também é mostrado como compor em uma árvore bi-variável, dois processos estocásticos independentes sendo que pelo menos um segue um MRM, e o outro um MGB ou outro MRM. Neste capítulo as abordagens desenvolvidas são usadas para avaliar uma opção de expansão de uma usina somente de açúcar, para produzir também etanol. O terceiro capítulo usa os resultados dos dois primeiros para avaliar a opção de alternância de produção existente nas usinas flexíveis de processamento de cana de açúcar. Estas podem alternar a produção entre açúcar ou etanol, e esta flexibilidade tem valor substancial para a usina. O capítulo avalia esta opção real usando a abordagem por árvore bi-variável combinando dois processos de Reversão à Média correlacionados para os preços dos dois produtos passíveis de serem produzidos. Esta modelagem é então comparada aos resultados de uma avaliação por simulação, os quais confirmam a convergência dos dois métodos. Também é analisada a sensibilidade do valor da opção real de alternância à correlação dos dois processos estocásticos. Finalmente a tese conclui com um resumo geral sobre a importância da escolha correta do processo estocástico que modela as incertezas envolvidas na avaliação por opções reais e sugere futuras linhas de pesquisa, tal como a parametrização de processos com saltos.

\section{Palavras Chave}

Opções Reais; Processos Estocásticos; Reversão à Média; Árvore Binomial; Árvore bi-variável; Processos Estocásticos de Dois Fatores; Bio-Combustíveis; Indústria de Etanol. 


\section{Abstract}

Bastian Pinto, Carlos de Lamare; Brandão, Luiz Eduardo Teixeira (Advisor). Real Options Modeling with Mean Reversion Processes in Discrete-Time: An Application in the Brazilian Ethanol Industry. Rio de Janeiro, Doctoral Dissertation, p. 164 - Departamento de Administração de Empresas, Pontifícia Universidade Católica do Rio de Janeiro

This dissertation covers the theme of stochastic uncertainties modeling with mean reversion, and its applications in real options valuation. The use of alternate stochastic processes other than random walk, or Geometric Brownian Motion (GBM), usually does not have analytical closed solutions for valuing contingent claims and therefore numerical approaches must be used. The dissertation is divided in three main chapters that cover this theme. In the first of these, after a discussion on the validity of stochastic process, the most widely known Mean Reversion Models (MRM) are presented: four single factor processes, one arithmetic and three geometric, and a two factor process. For all of these we show or develop the discrete process, the expression for the expected value and estimation of parameters from historical data. This last point is fundamental since generally only historical data is available for uncertainties involved in most real options applications. The chapter also estimates parameters of the main models presented for data from prices of ethanol and sugar paid to producers in the State of São Paulo. The second of these chapters deals with lattice modeling as a discrete method for approaching mean reversion processes. This approach allows the valuation of contingent claims written on a variable whose value follows this stochastic behavior. Lattice modeling is already a classic approach used in countless papers and originates from the methodology developed by Cox, Ross and Rubinstein (1979). But this latter method only fits variables showing an MGB dynamic, excluding the whole range of assets for which an autoregressive process is a better description of their price behavior. Two mean reversion lattice models are then explained. Also shown is an approach allowing the composition of a bivariate lattice with two independent yet correlated stochastic processes, of which 
one is a MRM and the other either an MGB or another MRM. These approaches are then used to value an expansion option available to a sugar producing plant to also produce ethanol. The third chapter uses the methodologies developed in the first two to value the switch option embedded in Brazilian sugarcane flexible plants. These can switch production from one output to another (ethanol and sugar) and this flexibility has significant value. The chapter values this real option using the bi-variate lattice approach, combining two correlated MRMs for both prices of the possible output products. This modeling is then compared to the results of a simulation method, which confirms the convergence of both results. We also analyze the sensibility of the option switch value to the correlation of both stochastic processes. Finally the dissertation concludes on the importance of the correct choice of the stochastic process when modeling the uncertainties involved in real options valuation, and suggests further research in the same line, such as parameterization of jump processes.

\section{Keywords}

Real Options; Stochastic Processes; Mean Reversion; Binomial Lattice; BiVariate Lattice; Two Factor Stochastic Processes; Bio-fuels; Brazilian SugarEthanol industry. 


\section{Sumário}

1 Introdução 18

1.1. Modelagem de Processos Estocásticos por Reversão À Média 19

1.2. Modelagem de Opções Reais por Árvores Binomiais 20

1.3. Aplicação de Opções Reais na Indústria Brasileira de Etanol 21

1.4. Organização da Tese 22

2 Processos Estocásticos de Reversão à Média para Aplicação em Opções Reais 24

$\begin{array}{ll}\text { Resumo } & 24\end{array}$

2.1. Introdução 24

2.2. Processos Estocásticos e seu Uso em Aplicações de Opções Reais 26

2.3. Determinação da Validade do Processo Estocástico 29

2.4. Modelos de Reversão à Média de Fator Único 32

2.4.1. Modelo Aritmético de Ornstein-Uhlenbeck 32

2.4.1.1. Média e Variância 33

2.4.1.2. Discretização do Modelo 33

2.4.1.3. Estimação de Parâmetros 34

2.4.1.4. Simulação Neutra ao Risco 36

2.4.2. Modelos Geométricos de Reversão à Média 37

2.4.2.1. O Modelo de Dixit \& Pindyck (1994) 37

2.4.2.1.1. Média e Variância 38

2.4.2.1.2. Discretização do Modelo 40

2.4.2.1.3. Estimação de Parâmetros 40

2.4.2.1.4. Simulação Neutra ao Risco 42

2.4.2.2. O Modelo 1 de Schwartz (1997) 43

2.4.2.2.1. Média e Variância 43

2.4.2.2.2. Discretização do Modelo 45

2.4.2.2.3. Estimação de Parâmetros 46 
2.4.2.2.4. Simulação Neutra ao Risco 47

2.4.2.3. O Modelo Dias/Marlim (1999) 48

2.4.2.3.1. Média e Variância 49

2.4.2.3.2. Discretização do Modelo 49

2.4.2.3.3. Estimação de Parâmetros $\quad 50$

2.4.2.3.4. Simulação Neutra ao Risco 52

2.4.3. Discussão acerca das limitações dos Modelos apresentados de Reversão à Média de Fator Único 52

2.5. Modelos de dois Fatores com Reversão à Média 54

2.5.1. O modelo de dois fatores de Schwartz e Smith (2000) 57

2.5.1.1. Equação diferencial do modelo 59

2.5.1.2. Média e Variância $\quad 59$

2.5.1.3. Discretização do modelo 60

2.5.1.4. Estimação de Parâmetros 62

2.5.1.4.1. Derivação dos valores iniciais dos dois fatores do processo 63

2.5.1.4.2. Primeiro caso: considera-se que $\sigma_{y}=0 \quad 63$

2.5.1.4.3. Segundo caso: o processo MAB é conhecido 65

2.5.1.4.4. Terceiro caso: Caso geral, apenas $S_{t}$ é observado 66

2.5.1.5. Simulação Neutra ao Risco 68

2.6. Resultados da modelagem de preços de açúcar e etanol com os modelos descritos 69

2.6.1. Dados utilizados 69

2.6.2. Determinação da Validade do Processo Estocástico 71

2.6.3. Resultados 73

2.7. Conclusões e sugestões $\quad 79$

2.8. Referências Bibliográficas 80

3 Árvores Binomiais para Aproximação de Movimento de Reversão à Média, para uso em Opções Reais 83

Resumo 83

3.1. Introdução: árvores binomiais recombinantes para avaliação de opções reais 83

3.2. Aproximação binomial para movimento de reversão à média 85 
3.2.1. Modelo de reversão à média censurado de Nelson e Ramaswamy (1990)

3.2.2. Aproximação binomial para modelo não censurado de reversão à média

3.2.3. Convergência dos dois modelos de árvore binomial para reversão à média 92

3.3. Modelos de árvore bi-variável com reversão à média 98

3.3.1. Modelo bi-variável composto de um MGB e um MRM 99

3.3.2. Modelo bi-variável composto de dois processos MRM 103

3.4. Avaliação de opção de expansão de usina refinadora de açúcar em destilaria flexível de etanol 105

3.4.1. Metodologia de avaliação da opção real de expansão 106

3.4.2. Modelagem e resultados 109

3.5. Conclusões 112

3.6. Referências bibliográficas 112

Anexo 3.1 Derivação dos valores de subida e descida e probabilidade de subida no modelo binomial para MRM não censurado

Anexo 3.2 Derivação do modelo censurado de Nelson e Ramaswamy (1990) para reversão à média

4 Flexibilidade como fonte de valor na produção de combustíveis alternativos: o caso do etanol brasileiro

4.1. Introdução 119

4.2. A indústria Brasileira de Etanol e Açúcar 121

4.3. Modelagem estocástica dos preços de etanol e açúcar 123

4.3.1. Aproximação binomial para processos de reversão à média 128

4.3.2. Transformação em um processo neutro a risco 130

4.3.3. Modelagem discreta bi-variável de processo de reversão à média

4.4. Metodologia de avaliação de opção de alternância 134

4.4.1. Estimação dos parâmetros dos processos estocásticos 134

4.4.2. Metodologia do modelo de avaliação da opção 
4.6. Resultados

4.6.1. Resultados do modelo de árvore bi-variável 141

4.6.2. Comparação com resultados de modelo de simulação 142

4.6.3. Comparação com resultados assumindo a modelagem dos preços por MGB

4.6.4. Sensibilidade do resultado à correlação entre os processos de preços

4.7. Conclusões 146

4.8. Referências bibliográficas 147

Anexo 4.1: Transformação do processo estocástico para o In (Preço) 150

Anexo 4.2: Ajustamento da media de longo prazo para um processo

neutro a risco

Anexo 4.3: Estimação de parâmetros em modelo de reversão à média

5 Conclusões e Recomendações para Futuras Pesquisas

6 Referências Bibliográficas Consolidadas 


\section{Lista de figuras}

Figura 2.1. Plotagem modelo Schwartz e Smith, com $\times 0>0 \quad 61$

Figura 2.2. Plotagem modelo Schwartz e Smith, com $\times 0<0 \quad 61$

Figura 2.3. Preços etanol e açúcar CEPEA 70

Figura 2.4. Preços etanol e açúcar deflacionados pelo IGP-DI -

CEPEA $\quad 70$

Figura 2.5. razão da variância $R k$ para diferentes valores de lag k etanol e açúcar $\quad 72$

Figura 2.6. Variância verificada e estimada para Etanol nominal $\quad 77$

Figura 2.7. Variância verificada e estimada para Açúcar nominal 78

Figura 3.1. Nó binomial 90

Figura 3.2. Nó binomial do processo OU 91

Figura 3.3. Árvores binomiais modelos censurado e não censurado, com S0 = $75 \quad 94$

Figura 3.4. Exemplo de árvore MRM com os dois modelos com S0 = $25 \quad 97$

Figura 3.5. Nó da ramificação bi-variável 99

Figura 3.6. Nó bi-variável censurado de um MAB seguido de um $\begin{array}{ll}\text { MRM } & 101\end{array}$

Figura 3.7. Nó bi-variável censurado de dois MRMs 104

Figura 3.8. Fluxos de Caixa Mensais e Razão dos Fluxos de Caixa Etanol/Açúcar $\quad 107$

Figura 3.9. Árvores da variável RE/A para processos MGB e MRM 110 Figura 3.10. Árvores da variável RE/A para processo conjunto MGB e MRM

Figura 4.1. Razão da Variância para diferentes valores de retardo 127

Figura 4.2. Nó de ramificação binomial 128

Figura 4.3. Nó da árvore bi-variável $\quad 131$

Figura 4.4. Sequência do nó marginal-condicional para duas commodities

Figura 4.5. Série de preços de etanol e açúcar deflacionados por 
IGP-DI

Figura 4.6. Regressão para determinação dos parâmetros do processo estocástico do etanol

Figura 4.7. Regressão para determinação dos parâmetros do processo estocástico do açúcar

Figura 4.8. Árvore de processo neutro à risco para açúcar $\quad 140$

Figura 4.9. Árvore de processo neutro à risco para etanol 140

Figura 4.10. Projeções de preços de açúcar (ajustada ao risco) 144

Figura 4.11. Projeções de preços de etanol (ajustada ao risco) 144

Figura 4.12. Valor da opção de alternância em função da correlação 


\section{Lista de tabelas}

Tabela 2.1. Processos estotásticos mais usuais

Tabela 2.2. Valores críticos assintóticos de teste t de Raiz Unitária.

Sem tendência temporal

Tabela 2.3. Valores críticos assintóticos de teste $t$ de Raiz Unitária.

Com tendência temporal

30

Tabela 2.4. Modelagem como MGB e como MRM

74

Tabela 2.5. Modelagem como reversão à média com drift (caso 1 -

Schwartz \& Smith)

Tabela 2.6. Modelagem como reversão à média com IGP-DI (caso 2

- Schwartz \& Smith)

Tabela 2.7. Modelagem como reversão à média e MAB (caso 3 -

Schwartz \& Smith)

76

Tabela 2.8. Sensibilidade da volatilidade $\sigma_{x}$, a correlação $\rho_{x y}$ entre os fatores $x$ e $y$

Tabela 3.1. Sensibilidade para os dois modelos em erro $\%(\Delta \%)$ de $E[x]$ e $\sigma^{2}[x]$

Tabela 4.1. Resultados da regressão para preços deflacionados de etanol e açúcar.

Tabela 4.2. Parâmetros estocásticos para etanol e açúcar

Tabela 4.3. Valor presente dos fluxos de caixa operacionais dos casos base determinísticos

Tabela 4.4. Comparação dos resultados por reverão à média vrs MGB 


\section{Lista de Siglas e Abreviaturas}

CALL - Opção de Compra

CAPM - Capital Asset Princing Model

D\&P - Dixit \& Pindyck

FCL - Fluxo de Caixa Livre

IGP-DI - Índice Geral de Preços - Disponibilidade Interna

MAB - Movimento Aritmético Browniano

MGB - Movimento Geométrico Browniano

MRM - Movimento de Reversão à Média

OU - Ornstein Uhlenbeck

PIB - Produto Interno Bruto

TOR - Teoria das Opções Reais

VPL - Valor Presente Líquido 